\title{
Design of ECG Signal Acquisition and Processing Circuit
}

\author{
Fang Yuan ${ }^{1 a^{*}}$, Weikang $\mathrm{Ou}^{1, \mathrm{~b}}$ and Wei Jiang ${ }^{1, \mathrm{c}}$ \\ ${ }^{1}$ State Key Laboratory Breeding Base of Nuclear Resources and Environment, East China Institute \\ of Technology, Nanchang, Jiangxi, China \\ afyuan@ecit.cn, b455302348@qq.com, cw186@163.com
}

Keywords: ECG; Acquisition; Processing; Circuit.

\begin{abstract}
This paper designs an ECG signal acquisition and processing circuit. The circuit includes preamplifier circuit, a high-pass filter circuit, a low-pass filter circuit, $50 \mathrm{~Hz}$ frequency filter circuit, the main amplifier circuit. Experiments show that it can effectively filter all kinds of interference in the ECG signal. The circuit can realize the acquisition and processing of ECG signal, which has the characteristics of low cost and easy operation.
\end{abstract}

\section{Introduction}

ECG is a weak signal and it is easy to interference by external noise. Therefore, the key of getting accurate ECG signal is how to remove noise. Currently, Most of scholars study how to filter baseline drift, $50 \mathrm{~Hz}$ frequency interference and high frequency noise of ECG.Research methods and focus are different. Among them, more research is software algorithm of the ECG signal acquisition and processing. However, many ECG signal processing algorithms mainly achieve the desired treatment effect through the simulation method. Timeliness and usefulness are weak and limited to hardware resource conditions. It is difficult to achieve in the specific circuit.

The circuit of design ECG signal acquisition and processing combines the characteristics of ECG.It designs the preamplifier circuit, high-pass filter circuit, low-pass filter circuit, $50 \mathrm{~Hz}$ frequency trap circuit, main amplifier circuit. It can solve ECG acquisition and interference problems and reduce the impact of external interference signals to the ECG signal acquisition. It can effectively filter out the interference signal and achieve more accurate ECG signal.

\section{Preamplifier Circuit Design}

The core chip of preamplifier circuit design is AD620 chip. The chip input offset voltage is $50 \mu \mathrm{V}$ and Maximum input offset drift is $0.6 \mu \mathrm{V} / \mathrm{C}$. The common mode rejection ratio is $100 \mathrm{db}$ and the input voltage noise is $9 \mathrm{nV} / 1 \mathrm{kHz}$. The magnification is 10 and it can satisfy low noise and low drifts requirements of the preamplifier [1-3].

It can be measured a variety of gain required resistance. The resistance is equal to $5.489 \mathrm{k} \Omega$ when the design of magnification is 10 times. Preamplifier circuit includes the right leg drive circuit and the shield drive components in addition to AD620.

\section{High-pass Filter Circuit}

High-pass filter cutoff frequency is generally not more than $0.05 \mathrm{~Hz}$ in order to remove the DC component of the ECG. The high-pass cutoff frequency is set at $0.03 \mathrm{~Hz}$ to avoid errors affect the accuracy of the device. The high-pass filter circuit diagram is shown in Fig. 1 [4-5]. 


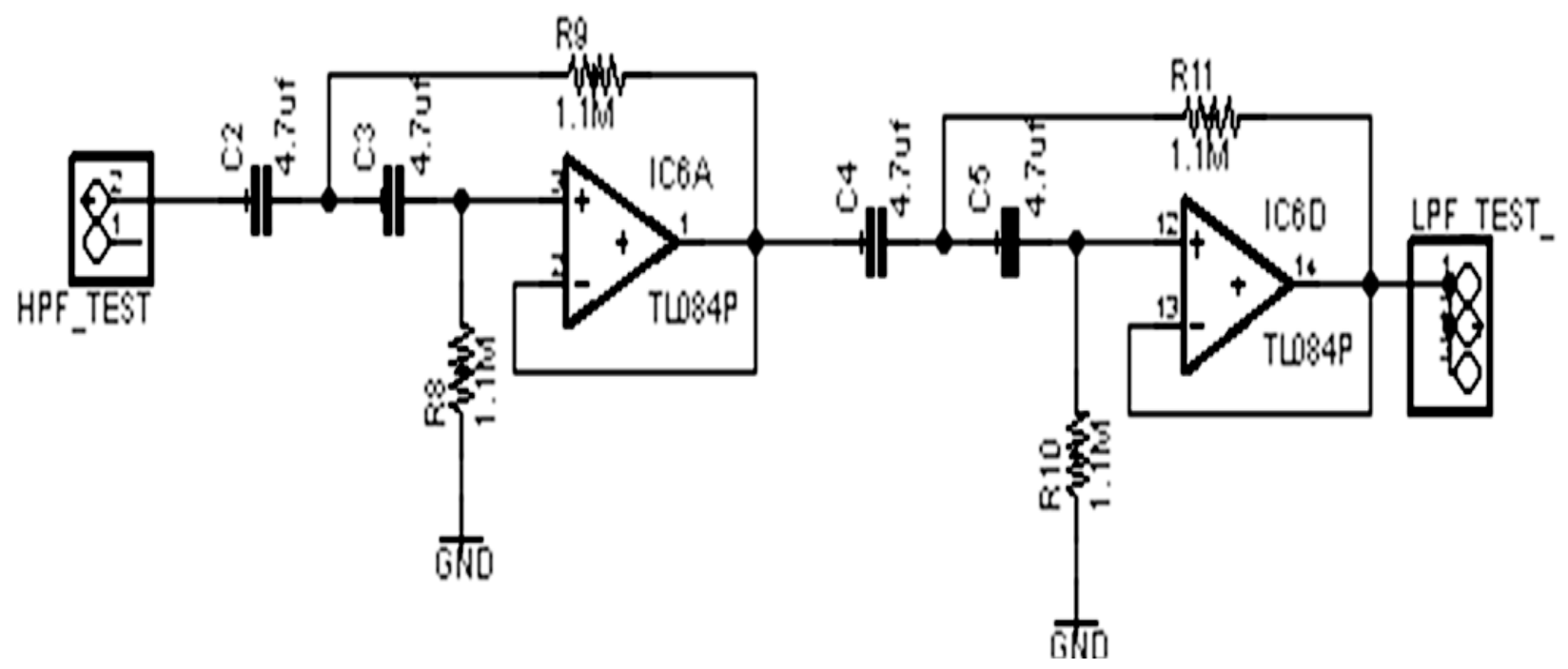

Figure 1. High-pass filter circuit

\section{Low-pass Filter Circuit}

The frequency of ECG is below $100 \mathrm{~Hz}$, so it is necessary to filter out high-frequency interference signals through a low pass filter circuit. The low-pass filter circuit diagram is shown in Fig. 2.

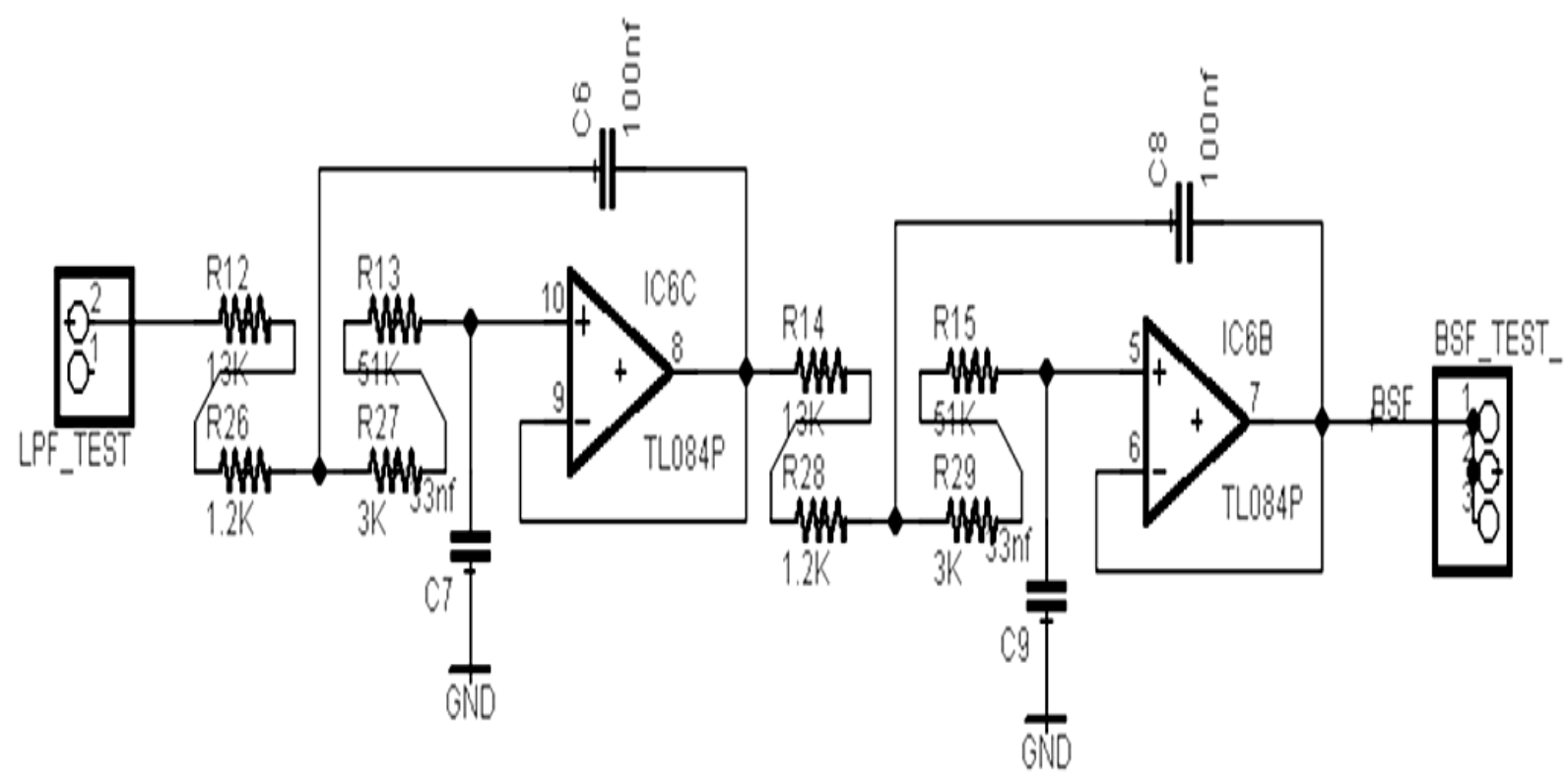

Figure 2. Low-pass filter circuit

\section{Hz Frequency Filter Circuit}

Preamplifier circuit has a strong inhibitory effect for common mode interference. But some frequency interference effect ECG signal by differential mode and the frequency is within the frequency band of the ECG signal. Therefore, it must be specifically filtered out. The design of circuit is a dual-T active band-stop filter. Bandwidth band-stop filter becomes narrower, the higher the quality factor Q. The inhibition selectivity of filter is better. However, it may not reach the center frequency of $50 \mathrm{~Hz}$ because of the accuracy error of the device. So, the $\mathrm{Q}$ value of circuit designs of 
$50 \mathrm{~Hz}$ frequency filter circuit can be adjusted. The $50 \mathrm{~Hz}$ frequency filter circuit diagram is shown in Fig. 3.

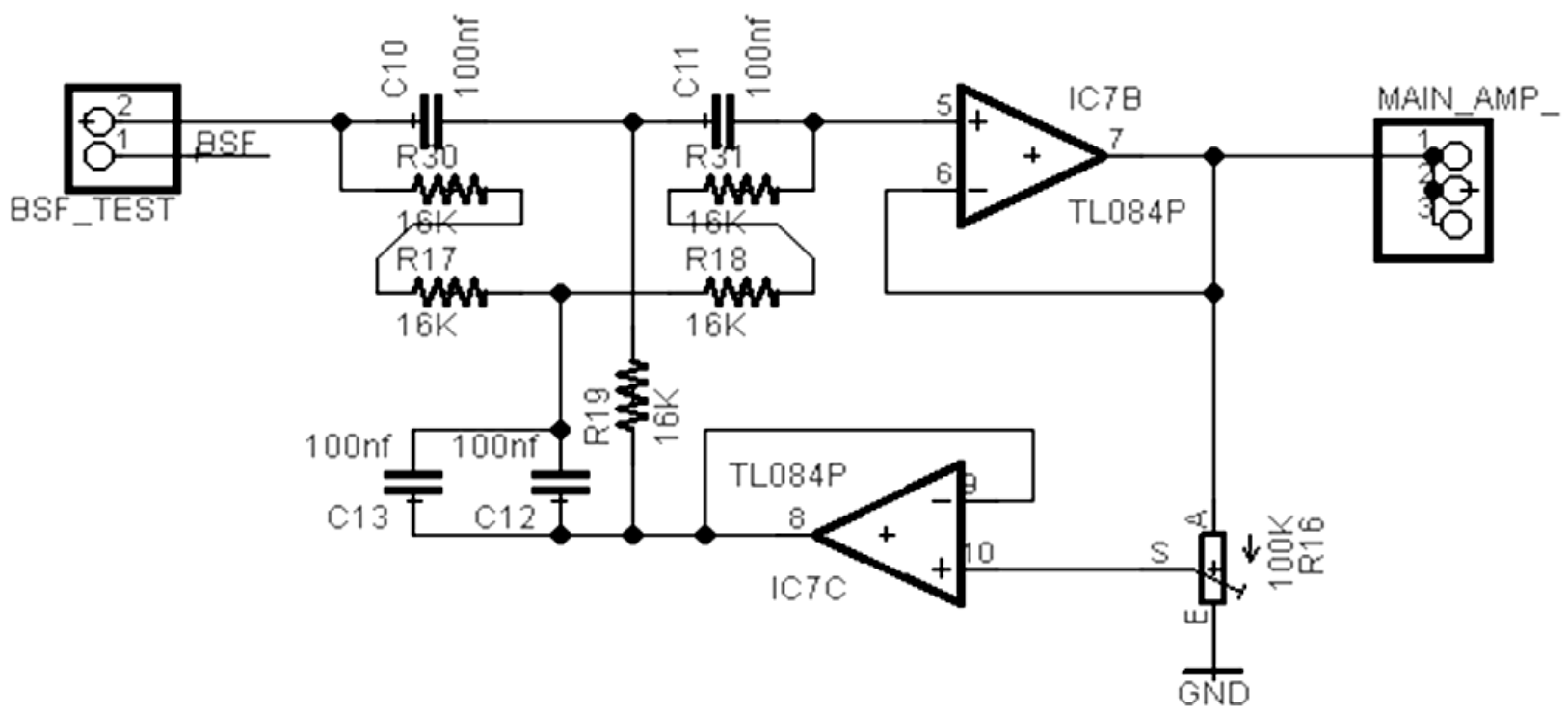

Figure 3. $50 \mathrm{~Hz}$ frequency filter circuit

\section{Main Amplifier Circuit}

Since the amplitude of the ECG signal is very small, generally only $1 \mathrm{mv}$, while the analog to digital conversion system chip voltage input range is $0 \sim 5 \mathrm{~V}$. Therefore, it is not enough only by the preamplifier 10 times magnification.it need to add a main amplifier circuits to improve the entire ECG amplifier gain. Since the pre-amplifier has been completed ECG 10 times. Amplifier circuit using two zoom mode to avoid high magnification unipolar lead to waveform distortion, the first stage to complete the 10 times magnification, the second stage to complete the 10-200 times adjustable amplification. The total gain of the system may be in the 100-2000 times by adjusted to meet the post-processing circuit voltage requirements [6-10]. The main amplifier circuit diagram is shown in Fig. 4.

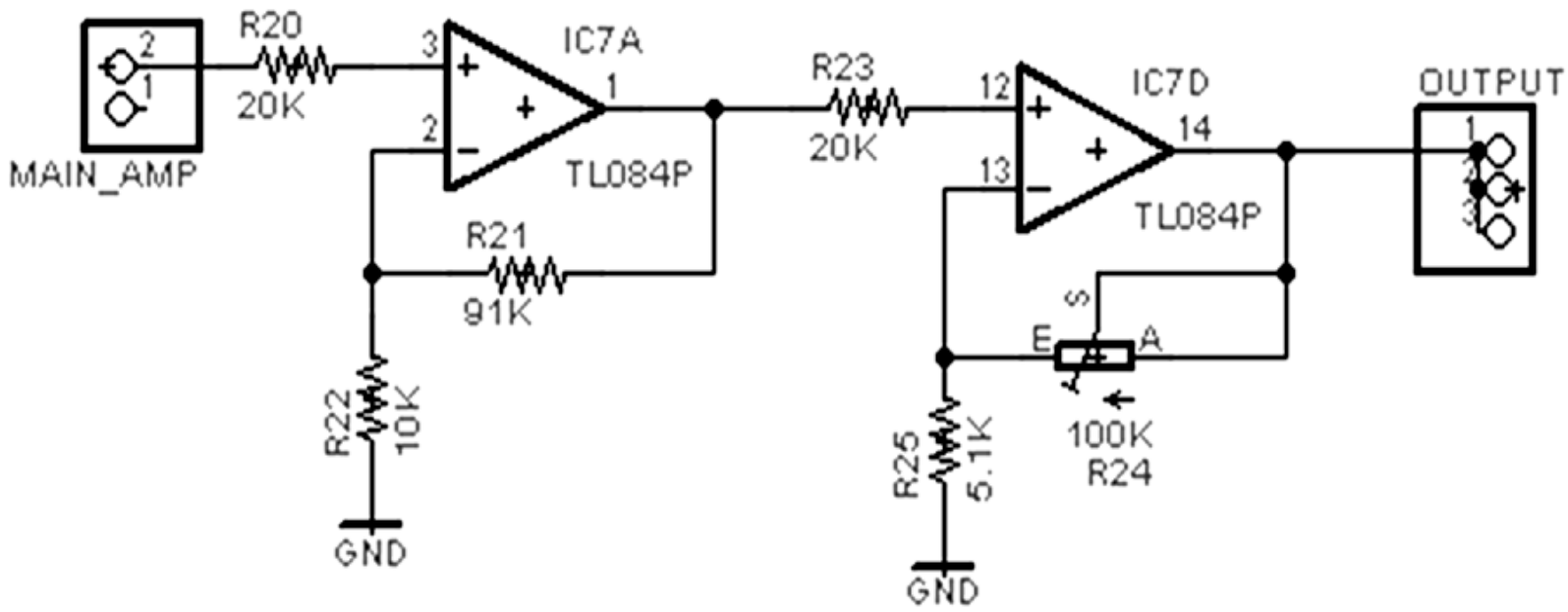

Figure 4. Main amplifier circuit 


\section{Summary}

It introduces an ECG signal acquisition and processing circuit. The circuit includes preamplifier circuit, a high-pass filter circuit, a low-pass filter circuit, $50 \mathrm{~Hz}$ frequency filter circuit, the main amplifier circuit. The circuit can effectively solve the ECG signal acquisition and interference problems, can effectively filter all kinds of interference in the ECG signal. It can get precise acquisition and processing ECG.

\section{Acknowledgements}

The science and technology support program of Jiangxi province (No.20141BBG70079) and the soft science research project of Jiangxi province (20151BBA10053) and college of humanities and social science research projects in Jiangxi province (GL1507) and Jiangxi education planning project (15YB041) and technology research project of Jiangxi education department (GJJ151534) (GJJ151543) and open fund of state key laboratory breeding base of nuclear resources and environment (No. NRE1413) supported this work.

\section{References}

[1] LIANG ZH H,GU Y,YANG CH L, Remote EEG-ECG monitoring system based on Android platform. Foreign Electronic Measurement Technology. 33 (2014) 91-94.

[2] UENO A,AKABANE Y,KATO T, Capacitive sensing of electrocardiographic potential through cloth from the dorsal surface of the body in a supine position: A preliminary study Biomedical A preliminary study. Biomedical Engineering, IEEE Transactions on. 54(2007)759-766.

[3] BAEK H J,CHUNG G S,KIM K K,A smart health monitoring chair for nonintrusive measurement of biological signals. Information Technology in Bio-medicine,IEEE Transactions on. 16(2012) 150-158.

[4] ZHOU P,WANG F ,LIU M X, Design of capacitance-based non-contact ECG sensor.Instrument Technique and Sensor. 34(2013) 2226-2230.

[5] Carolyn H Declerck, Christophe Boone, On Feeling in Control: A Biological Theory for Individual Differences in Control Perception. Brain and Cognition. 62(2006) 143-176.

[6] ABDI H,WILLIAMS L J, Principal component analysis. Wiley Interdisciplinary Reviews: Computational Statistics. 2(2010) 433-459.

[7] Bhyri, Channappa, Hamde, S.T, ECG feature extraction and diseas diagnosis. Journal of Medical Engineering and Technology. 35(2011) 354-361.

[8] Bert Uwekohler,The Prineiples of software QRS detection. IEEE Engineering in Medicine and Biology Magazine. 21(2002) 42-50.

[9] Information on http://www.mamicode.com

[10] Information on http://www.eeworld.com.cn 